\title{
Crystallography online by the Bilbao Crystallographic Server: new computer tools for the study of layer and multi-layer materials
}

\author{
G. de la Flor ${ }^{1}$, E. Tasci ${ }^{2}$, L. Elcoro ${ }^{3}$, G. Madariaga ${ }^{3}$, J. M. Perez-Mato ${ }^{3}$, Y. E. Kitaev, R. Evarestov ${ }^{5}$, M. I. Aroyo ${ }^{3}$ \\ ${ }^{1}$ Institute of Applied Geosciences, Karlsruhe Institute of Technology, Karlsruhe (Germany), \\ ${ }^{2}$ Department of Physics Engineering, Hacettepe University, Ankara (Turkey), \\ ${ }^{3}$ Departamento de Física de la Materia Condensada, Universidad del País Vasco UPV/EHU, Leioa, (Spain), \\ ${ }^{4}$ Laboratory of Quantum-size Heterostructures, Ioffe Institute, Saint Petersburg (Russia), \\ ${ }^{5}$ Institute of Chemistry, Saint Petersburg State University, Saint Petersburg (Russia) \\ gemma.delaflor@kit.edu
}

The interest in layered and multi-layered materials such as graphene and van der Waals crystals, e.g. the transition metal dichalcogenide crystal family, is constantly growing owing to their interesting properties and possible technological applications. The symmetry of single monolayers can be described by the so-called layer groups, which are three-dimensional crystallographic groups with two-dimensional translations. Due to the arising interest in these type of materials, new programs dedicated to the study of materials with layer and multilayer symmetry have been developed and implemented in the Bilbao Crystallographic Server (www.cryst.ehu.es) [1,2]. The server is in constant improvement and development, offering free of charge tools to study an increasingly number of crystallographic systems which now also includes the ones with layer symmetry.

The section dedicated to Subperiodic groups in the Bilbao Crystallographic Server gives access to the layer groups databases which contains the basic crystallographic information of the 80 layer groups (generators, general positions, Wyckoff positions and maximal subgroups) [3] and the Brillouin zone and k-vectors tables that form the background and classification of the irreducible representations of layer groups which can be calculated with one of the programs available in the server. More sophisticated programs to identify the layer symmetry of periodic sections and to describe the electronic structure and surface states of crystals [4] are also available. The symmetry relations between the localized state (atomic displacements) and extended states (phonon, electrons) over the entire Brillouin zone can also be calculated. The utility of the available applications will be demonstrated by illustrative examples.

[1] Aroyo, M. I., Perez-Mato, J. M., Capillas, C., Kroumova, E., Ivantschev, S., Madariaga, G., Kirov, A. \& Wondratschek H. (2006). Z. Krist. 221, $15-27$.

[2] Aroyo, M. I., Perez-Mato, J. M., Orobengoa, D., Tasci, E., de la Flor, G. \& Kirov A (2011). Bulg. Chem. Commun. 43(2), $183-197$.

[3] International Tables for Crystallography Vol. E: Subperiodic-Groups (2002), edited by Kopský and D.B. Litvin. Dordrecht: Kluwer Academic Publischers.

[4] de la Flor, G., Orobengoa, D., Evarestov, R. A., Kitaev, Y. E., Tasci, E. \& Aroyo M. I. (2019). J. Appl. Cryst. 52, 1214-1221

Keywords: Bilbao Crystallographic Server, symmetry, layer groups, layer and multilayer materials 